\title{
Therapy with bone-modifying agents in cancer patients: efficacy versus adverse events in dental medicine
}

\author{
Olimpia Bunta ${ }^{1}$, Iulia Najette Crintea ${ }^{2,3}$, Andreea Pop ${ }^{4}$, Adina Nemes ${ }^{6,7}$, Mariana Pacurar ${ }^{5}$ \\ ${ }^{1}$ Orthodontics Department, "Iuliu Hatieganu" University of Medicine and Pharmacy, Cluj-Napoca, Romania \\ 2"Victor Babes" University of Medicine and Pharmacy, Timisoara, Romania \\ 3"Pius Brinzeu" Timis County Emergency Clinical Hospital, Timisoara, Romania \\ ${ }^{4}$ Oral Rehabilitation Department, "Iuliu Hatieganu“ University of Medicine and Pharmacy, \\ Cluj-Napoca, Romania \\ ${ }^{5}$ Orthodontics Department, "G.E. Palade" University of Medicine and Pharmacy Science and Technology, \\ Tg. Mures, Romania \\ ${ }^{6}$ Medical Oncology Department, "Iuliu Hatieganu" University of Medicine and Pharmacy, \\ Cluj-Napoca, Romania \\ 7"Prof. Dr. Ion Chiricuta" Oncology Institute, Cluj-Napoca, Romania
}

\begin{abstract}
Bone metastases affect a large number of oncological patients and can determine complications that can impact the quality of life of our patients and further translate into lessen treatment results and overall survival. Treatment with bisphosphonates (BP) has proven its efficacy in preventing the development of the complications related to bone metastases. BP are well tolerated in general but severe adverse events can occur, like osteonecrosis of the jaw (ONJ). Besides ONJ there are other dental and maxillofacial complications determined by BP, complications that by elementary preventive measures can be avoided.
\end{abstract}

Keywords: bone-modifying agents, bisphosphonates, oral cancer, bone metastases, dental medicine, osteonecrosis of the jaw, dental implants

\section{INTRODUCTION}

Bone metastases commonly affect patients with prostate, breast, kidney and lung cancer and also patients with multiple myeloma and can determine skeletal complications known as skeletal related events (SRE) that impair the quality of life and survival of oncological patients. SRE are represented by spinal cord compression, pathological fracture, the need for radiotherapy or surgery to bone and hypercalcaemia [1]. Approximately 40\% of patients with lung cancer and bone metastases will present a SRE and their median survival ranges from 6 to 12 months [2]. In patients with breast cancer and bone metastases SRE were observed in more than $50 \%$ of patients and median survival was 7 months in patients that developed SRE versus 16 months in patients with no SRE based on the results of a study that included 36,000 patients with breast cancer [3]. The treatment of SRE implies prolonged hospitalization and increased costs and can be represented by external beam radiation therapy, radioisotopes, endocrine therapy, chemotherapy, targeted therapy, therapy with bone-modifying agents and even surgery [1].

\section{EFFICACY OF BISPHOSPHONATES (BP) IN BONE METASTASES}

BP are analogues of pyrophosphate that administered in patients with bone metastases reduce skeletal morbidity and relieve bone pain [4-6]. BP 
inhibit osteoclast activity determining decreased bone resorption and increased mineralization, primarily at active remodeling sites [4].

$\mathrm{BP}$ are recommended in patients with breast cancer and bone metastases, in patients with castration resistant prostate cancer and bone metastases, in patients with lung cancer, kidney cancer and other solid tumors with bone metastases and in patients with multiple myeloma [7]. Zoledronic acid is the most commonly used BP in clinical practice but there are also other BP approved in cancer patients [1].

Administration of zoledronic acid in patients with breast cancer and bone metastases reduced the risk of developing SRE by $20 \%$ when compared to pamidronate $(p<0.05)$ [8]. Saad and colleagues showed in a placebo-controlled study that included 643 patients with castration resistant prostate cancer that administration of zoledronic acid determined fewer SRE (33\% vs. $44 \%$ in the placebo arm, $p=0.021$ ), a longer time to the first SRE and improvement in bone pain [9]. When administered in patients with other solid tumors zoledronic acid also reduces the risk of SRE (38\% zoledronic acid arm vs. $47 \%$ placebo arm) and prolongs the time to the first SRE (230 days zoledronic acid arm vs. 163 days placebo arm) [10].

\section{ADVERSE EVENTS OF BISPHOSPHONATES IN DENTAL MEDICINE}

Treatment with BP is well tolerated and the most common adverse events observed are acutephase reactions, hypocalcaemia, renal dysfunction, arthralgias and ONJ [11-13]. Besides these adverse events there are other dental and maxillofacial complications that can occur in patients with prolonged administration of BP such as pain, swelling of soft tissues, suppuration, ulceration, sinus tract infection [14-16], extraction socket persistence [17], dental implant failure, periodontal pockets, tooth mobility, delay in teeth eruption and prolonged orthodontic treatments with incomplete space closure that can affect the quality of life of these patients and lead to complications that can determine treatment delays, and subsequently alter overall survival.

ONJ is defined as an area of exposed bone in the maxillofacial region that did not heal within 8 weeks after identification by a health care provid- er, in a patient who was receiving or had been exposed to a BP and had not had radiation therapy to the craniofacial region and can be observed in approximately $1 \%-10 \%$ of the patients that receive $\mathrm{BP}$, especially in patients that receive monthly BP iv and have preexisting dental problems $[13,18$ 24]. The susceptibility of the jaws to ON may be determined by several characteristics of the anatomy and physiology of the bone. Their increased prevalence in the maxilla and in the mandible compared to other bones, is assumed to be due to BP preference to deposit in high turnover bones with increased remodeling properties, determined by the masticatory system physiology and particularities $[15,25,26]$.

The duration of treatment with BP, the dose used, the type of solid tumor and concurrent administration of other drugs such as Trastuzumab, glucocorticoids and antiangiogenic drugs may increase the incidence of ONJ [27,28]. The incidence of ONJ can be reduced by dental preventive measures such as dental consultation and orthopantomography and dental care if required before the initiation of BP. In a study that evaluated the importance of dental preventive measures in reducing the risk of ONJ Ripamonti and colleagues found that only $1.3 \%$ of the patients that underwent these measures developed ONJ versus 3.2\% of the patients that did not have a dental consultation before initiation of BP [29]. An interesting observation was made by Iuliis DE et al. in their study, according to which, based on their analysis of 200 solid tumor with bone metastases patients that underwent zoledronic acid treatment, after daily administration of vitamin D and calcium, recommendation to reduce alcohol intake and stop smoking, the results did not mention a single ONJ case after the 6 month assessment period [30].

Bone mineral density (BMD) measurements have a very important role in the assessment of bone quality. Nevertheless, the dual energy x-ray absorptiometry (DXA) does not always accurately predict the risks overtaken, in terms of bone quality, when using pharmacological products. Bone turnover, microarchitecture, mineralization, micro-damage and matrix/mineral composite, using different investigation techniques, are important tools in assessing bone quality in oncological patients treated with BP [31]. 
When assessing the bone mineral density (BMD) of the jaws, multiple studies have demonstrated that dental radiographs such as panoramic images, offer pertinent predictions in patients with BMD modifications, using the dental panoramic indices (mandibular cortical index-MCI, mental index-MI, panoramic mandibular index-PMI) $[32,33]$, but not always in correlation with other scheletal sites [33]. Another study showed that patients diagnosed with BP related ONJ, presented an increased mandibular inferior cortical bone thickness compared with patients treated with BP but without ONJ and also controls [34].

In dental practices, panoramic X-rays (as two-dimensional image) and CBCTs (as three dimensional-images), offer substantial information about BMD levels of oncological patients undergoing BP therapy. As risks of developing ONJ are higher in these patients, a pre BP treatment dental evaluation should be mandatory.

From a dental medicine point of view, the implications and complications of BP therapy in oncological patients are multiple. Prevention of oral pathologies, through improvement of oral hygiene, is the best possible way to reduce the risk of developing ONJ. Whenever oral pathologies do appear, these should be treated before beginning treatment with BP.

In a retrospective study conducted by Fung $\mathrm{P}$ et al. it was demonstrated that the risk of ONJ in jaws increases considerably with the BP treatment duration [35].

During BP treatment, tooth extraction especially is associated with a very high risk of ONJ [36,37]. A case report published in 2015, has showed the direct correlation between long intravenous administration of BP and tooth extraction with the occurrence of characteristic radiographic aspect of ONJ, in a multiple myeloma patient [38]. There have been studies which proved that antibiotic prophylaxis, 2-4 days before and 7-10 days after the procedure in association with antimicrobial mouth rinsing [27], reduces the risk of ONJ up to $0 \%$ [39].

Another dental procedure, bone surgery in or outside the context of tooth extraction, is considered to be highly associated with ONJ occurrence. Ghidini $\mathrm{G}$ et al. have demonstrated in their retrospective study that Er:YAG laser treatment or LLLT improve the outcomes of dental surgical therapy in oncological patients that undergo BP therapy [40]. The same conclusion was drawn by Vescopi P. et al. when studying their proposed surgical protocol using LLLT therapy in tooth extractions [41].

A surgical procedure in the oral cavity, dental implant positioning, is also influenced by the use of BP, as Yajima $\mathrm{N}$ et al. have published in their findings and showed increased cortical BMD directly correlated with duration of $\mathrm{BP}$ treatment [42]. A case report published in 2015 has drawn attention to the fact that metastatic breast cancer in patients treated with BP leads to the occurrence of metastatic cancer deposits in peri-implant sites, after precise histopathological analysis [43]. Also, in a study published in 2017 by Pogrel MA and Ruggiero SL, the authors proved the occurrence of a localized osteonecrosis around previously successfully inserted implants [44,45], without any modifications in the structure of the adjacent remaining teeth bone [44]. An interesting observation was made by Mozzati M. et al. in their research, which states the fact that the administration way of BP will also influence the occurrence degree of ONJ in oncological patients in need of dental implants. Based on their research and the American Association of Oral and Maxillofacial Surgeons, intravenous BPs contraindicates dental implant placement, compared with orally administered BPs which only imply caution when undergoing this surgical procedure [46].

There is also evidence of periodontal involvement preceding ONJ in oncological patients receiving BP. As Nicolatou-Galatis $\mathrm{O}$ et al. published in their study, the symptoms included pain, bleeding, fistula, swelling, pockets and tooth mobility, and lasted for 8-24 weeks before ONJ diagnosis [47].

When analyzing the effect of zoledronate on dental pulp stem cells and gingival fibroblasts, Pourgonabadi S et al. concluded in their study that this third generation BP has anti-proliferative and pro-apoptotic effects on the stem cells, thus having an unfavorable impact on regenerative potentials of the human body [48].

Of course, the mechanisms of ONJ formation are still being studied. In 2018, Cui P et al. have demonstrated the blocking of the osteocyte-osteoblast communication, thus new bone formation confirmed by bone histomorphometry, when administrating zoledronate systemically [49]. 
Following the same idea, Hernandez $\mathrm{M}$ et al. approached the same mechanism of osteoclast inhibition in children, emphasizing this interference of this phenomena in the temporary teeth exfoliation and eruption processes of permanent teeth, determining delays in tooth eruption patterns. Also, their study showed no reported ONJ cases in children treated with BP [50].

From an orthodontic point of view, the study conducted by Krieger $\mathrm{E}$ et al. demonstrated bone metabolism modifications, with consequences like tooth movement deceleration and therefor longer treatment duration, incomplete space closure and lack of root parallelism especially in crowding cases treated with extractions or initial spaces [51].

\section{CONCLUSIONS}

Treatment with BP has long time proven its efficacy in prevention of SRE in cancer patients with

\section{REFERENCES}

1. Coleman R, Body JJ, Aapro M, Hadji P, Herrstedt J. Bone health in cancer patients: ESMO clinical practice guidelines. Ann Oncol. 2014;25:iii124-iii37.

2. Decroisette $\mathrm{C}$, Monnet I, Berard $\mathrm{H}$ et al. Epidemiology and treatment costs of bone metastases from lung cancer: a French prospective, observational, multicenter study (GFPC 0601). J Thorac Oncol. 2011;6:576-82.

3. Coleman RE. Clinical features of metastatic bone disease and risk of skeletal morbidity. Clin Cancer Res. 2006;12(Suppl 20):6243s-9s.

4. Roelofs AJ, Thompson K, Ebetino FH et al. Bisphosphonates: molecular mechanisms of action and effects on bone cells, monocytes and macrophages. Curr Pharm Des. 2010;16:2950-60.

5. Wong R, Wiffen PJ. Bisphosphonates for the relief of pain secondary to bone metastases. Cochrane Database Syst Rev 2002;2:CD002068.

6. Coleman RE, McCloskey EV. Bisphosphonates in oncology. Bone. 2011;49:71-6.

7. Aapro M, Abrahamsson PA, Body JJ et al. Guidance on the use of bisphosphonates in solid tumours: recommendations of an international expert panel. Ann Oncol. 2008;19:420-32.

8. Rosen LS, Gordon D, Kaminski M et al. Long-term efficacy and safety of zoledronic acid compared with pamidronate disodium in the treatment of skeletal complications in patients with advanced multiple myeloma or breast carcinoma: a randomized, double-blind, multicenter, comparative trial. Cancer. 2003;98:1735-44.

9. Saad F, Gleason DM, Murray R et al. Zoledronic Acid Prostate Cancer Study Group. Long-term efficacy of zoledronic acid for the prevention of skeletal complications in patients with metastatic hormone-refractory prostate cancer. J Natl Cancer Inst. 2004; 96:879-82

10. Rosen LS, Gordon D, Tchekmedyian NS et al. Long-term efficacy and safety of zoledronic acid in the treatment of skeletal metastases in patients with non small cell lung carcinoma and other solid tumors: a randomized, phase III, double blind, placebo-controlled trial. Cancer. 2004;100:2613-21.

11. Lipton A, Fizazi K, Stopeck AT et al. Superiority of denosumab to zoledronic acid for prevention of skeletal-related events: a combined bone metastases but the positive effects can sometime be encumbered by multiple dental complications that impact the quality of life of our patients and systemic treatment results.

The doctors should be aware of the possibility of these complications occurrence and improuve the active prevention methods reduce the incidence of these adverse events.

In the same time, the patients who need an surgical treatment in dentistry (extraction) should inform the doctor about BP in medical history.

\section{Acknowledgement}

All authors have equal contributions. analysis of 3 pivotal, randomised, phase 3 trials. Eur $\mathrm{J}$ Cancer. 2012;48:3082-92.

12. Vomvas D, Vassiliou V, Papavasileiou D et al. Osteonecrosis of the jaw in a patient treated with ibandronate. JBUON 2008;13:441-2.

13. Tarassoff $P, C$ sermak K. Avascular necrosis of the jaw: risk factors in metastatic cancer patients. J Oral Maxillofac Surg. 2003;61:1238-9.

14. Nicolatou-Galitic O, Papadopoulou E, Sarri T et al. Osteonecrosis of the jaw in oncology patients treated with bisphosphonates: prospective experience of a dental oncology referall center. Oral Surg Oral Med Pathol Oral Radiol Endod. 2011;112:195-202.

15. Paulo S, Abrantes AM, Laranjo M et al. Bisphosphonate-related osteonecrosis of the jaw:specificities. Oncol Rev. 2014;8(2):44-9.

16. Wigler R, Steinbock N, Berg T. Oral cutaneous sinus tract, vertical root fracture, and bisphosphonate-related osteonecrosis: a case report. J Endod. 2013;39(8):1088-90.

17. Mawardi H, Treister N, Richardson P et al. Sinus tract - an early sign of bisphosphonate-associated osteonecrosis of the jaw? J Oral Maxillofac Surg. 2009;67(3):593-601.

18. Khosla S, Burr D, Cauley J et al. American Society for Bone and Mineral Research. Bisphosphonate-associated osteonecrosis of the jaw: report of a task force of the American Society for Bone and Mineral Research. J Bone Miner Res. 2007;22:1479-1491.

19. Mavrokokki A, Cheng A, Stein B, Goss AN. Nature and frequency of bisphosphonate-associated osteonecrosis of the jaws in Australia. J Oral Maxillofac Surg. 2007;65:415-423.

20. Woo S-B, Hellstein JW, Kalmar JR. Systematic review: Bisphosphonates and osteonecrosis of the jaws. Ann Intern Med. 2006;144:753-761.

21. Durie BGM, Katz M, Crowley J. Osteonecrosis of the jaw and bisphosphonates. N Engl J Med. 2005;353:99.

22. Hoff AO, Toth BB, Altundag $\mathrm{K}$ et al. Osteonecrosis of the jaw in patients receiving intravenous bisphosphonate therapy. Clin Oncol. 2006; 24:8528.

23. Van Poznak CH, Estilo C, Williams T et al. Osteonecrosis of the maxilla and mandible in patients with metastatic breast cancer. J Bone Miner Res. 2004;19:S1;S227. 
24. Migliorati CA, Epstein JB, Abt E, Berenson JR. Osteonecrosis of the jaw and bisphosphonates in cancer: a narrative review. Nat Rev Endocrinol. 2011;7:34-42.

25. Ikebe T. Pathophysiology of BRONJ: drug related osteoclastic disease of the jaw. Oral Sci Int. 2013;10:1-8.

26. Sarin J, DeRossi SS. Updates on bisphosphonates and potential pathobiology of bisphosphonate-induced jaw osteonecrosis. Oral Dis. 2008;14:277-85.

27. Fassio A, Bertoldo F, Idolazzi L et al. Drug-induced osteonecrosis of the jaw: the state of the art. Reumatismo 2017;69(1):9-15.

28. Pilanci KN, Alco G, Ordu $C$ et al. Is administration of Trastuzumab an independent risk factor for developing osteonecrosis of the jaw among metastatic breast cancer patients under zoledronic acid treatment? Medicine. 2015;24:1-5.

29. Ripamonti $\mathrm{Cl}$, Maniezzo M, Campa T et al. Decreased occurrence of osteonecrosis of the jaw after implementation of dental preventive measures in solid tumor patients with bone metastases treated with bisphosphonates. The experience of the National Cancer Institute of Milan. Ann Oncol. 2009;20:137-45.

30. Iuliis DE, Taglieri L, Amoroso L et al. Prevention of osteonecrosis of the jaw in patients with bone metastases treated with bisphosphonates. Anticancer Dis. 2014;34(5):2477-80.

31. Compston J. Bone quality: what is it and how is it measured? Arq Bras Endocrinol Metab. 2006;50(4):579-85.

32. Alasmari A, Lin SC, Daibart S et al. Bone microenvironmentmediated resistance of cancer cell to bisphosphonates and impact on bone osteocytes/stem cells. Clin Exp Metastasis. 2016; 33(6):563-88

33. Gulsahi A, Paksoy CS, Ozden S et al. Assessment of bone mineral density in the jaws and its relationship to radiomorphometric indices. Dentomaxillofac Rad. 2010;39:284-9.

34. Torres SR, Chen CS, Leroux BG et al. Mandibular inferior cortical bone thickness on panoramic radiographs in patients using bisphosphonates. Oral Surg Oral Med Pathol Oral Radiol Endod. 2015;119(5):584-92.

35. Fung P, Bedogni $G$, Bedogni A et al. Time to onset of bisphosphonate-related osteonecrosis of the jaws: a multicenter retrospective cohort study. Oral Dis. 2017;23(4):477-83.

36. Ribeiro NR, Silva Lde F, Santana DM et al. Bisphosphonate-related osteonecrosis of the jaw after tooth extraction. J Craniofac Surg. 2015;26(7):e606-8.

37. Utreja A, Almas K, Javed F. Dental extraction as a risk factor for bisphosphonate related osteonecrosis of the jaw in cancer patients: an update. Odontostamatol Trop. 2013;36(142):38-46.

38. Lee BD, Park MR, Kwon KH. Bisphosphonate-related osteonecrosis of the jaw in a multiple myeloma patient: a case report with characteristic radiograph features. Imaging Sci Dent. 2015; 45(3):199-203.

\section{Conflict of interest: none declared}

Financial support: none declared
39. Poxleitner $\mathrm{P}$, Engelhardt M, Schmelzeisen $\mathrm{R}$ et al. The prevention of medication-related osteonecrosis of the jaw. Dtsch Arztebl Int. 2017;114(5):63-9.

40. Ghidini G, Manfredi M, Giovannacci I et al. Medication-related osteonecrosis of the jaw: risk factors in patients under bisphosphonate versus patients under antiresorptive-antiangiogenic drugs. Minerva Stomatol. 2017;66(4):135-40.

41. Vescopi P, Giovannacci I, Merigo E et al. Tooth extractions in high-risk patients under bisphosphonate therapy and previously affected with osteonecrosis of the jaw: surgical protocol supported by low-level laser therapy. J Craniofac Surg. 2015;26(3):696-9.

42. Yajima N, Munakata M, Fuchigami K et al. Influence of bisphosphonates on implant failure rates and characteristics of postmenstrual woman mandibular jawbone. J Oral Implantol. 2017;43(5):345-9.

43. Favia G, Tempesta A, Limongelli L et al. Metastatic breast cancer in medication-related osteonecrosis around mandibular implants. Am J Case Rep. 2015;16:621-6.

44. Progrel MA, Ruggiero SL. Previously successful dental implants can fail when patients commence anti-resorptive therapy - a case series. Int J Oral Maxillofac Surg. 2018;47(2):220-2.

45. Giovannacci I, Meleti M, Manfredi M et al. Medication repated osteonecrosis of the jaw around dental implants: implant surgerytriggered or implant presence-triggered osteonecrosis? J Craniofac Surg. 2016;27(3):697-701.

46. Mozzati M, Arata V, Giacomello M et al. Failure risk estimates after dental implant placement associated with plasma rich in growth factor-Endoret in osteoporotic women under bisphosphonate therapy. J Craniofac Surg. 2015;26(3):749-55.

47. Nicolatou-Galitic O, Razis E, Galitis D et al. Periodontal disease preceding osteonecrosis of the jaw (ONJ) in cancer patients receiving antiresorptives alone or combined with targeted therapies: report of 5 cases and literature review. Oral Surg Oral Med Oral Pathol Oral Radiol. 2015;12096:699-706.

48. Pourgonabadi S, Mousavi SH, Tayarany-Najaran Z et al. Effect of zoledronate, a third-generation bisphosphonate, on proliferation and apoptosis of human dental pulp stem cells. Can J Physiol Pharmacol. 2017;3:1-8.

49. Cui P, Liu H, Sun J et al. Zoledronate promotes bone formation by blocking osteocyte-osteoblast communication during bone defect healing. Histol Histopathol. 2018;33(1):89-99.

50. Hernandez M, Phulpin B, Mansuy L et al. Use of new targeted cancer therapies in children: effects on dental development and risk of jaw osteonecrosis: a review. J Oral Pathol Med. 2017; 46(5):321-6.

51. Krieger $E$, Jacobs $C$, Walter $C$ et al. Current state of orthodontic patients under bisphosphonate therapy. Head Face Med. 2013;9:10. 